\title{
Dark matter and X-ray halo in early-type galaxies and clusters of galaxies
}

\author{
Takaya Ohashi \\ Department of Physics, Tokyo Metropolitan University, Hachioji, Tokyo 192-0397, Japan \\ email: ohashi@phys.metro-u.ac.jp
}

X-ray observations reveal extended halos around early-type galaxies which enable us to trace the dark matter distribution around the galaxies (see Mathews and Brighenti 2003 for a review). X-ray luminosities, $L_{\mathrm{X}}$ of massive early-type galaxies are $10^{40}-10^{42} \mathrm{erg}$ $\mathrm{s}^{-1}$ in $0.3-2 \mathrm{keV}$. The correlation plot between $L_{\mathrm{X}}$ and B-band luminosity $L_{\mathrm{B}}$ shows a large scatter in the sense that $L_{\mathrm{X}}$ varies by 2 orders of magnitudes for the same $L_{\mathrm{B}}$, in the brightest end $\left(\log L_{\mathrm{B}} \gtrsim 10.5\right)$. The amount of the X-ray hot gas in early-type galaxies is typically a few $\%$ of the stellar mass, in contrast to clusters of galaxies which hold $\sim 5$ times more massive gas than stars. Matsushita (2001) showed that X-ray luminous galaxies are characterized by extended X-ray halo with a few tens of $r_{e}$, similar to the scale of galaxy groups, so the presence of group-size potentials would be strongly linked with the problem of large $L_{\mathrm{X}}$ scatter.

Fukazawa et al. (2006) carried out extensive X-ray study of 53 early-type galaxies. They showed that gravitational mass profiles can be divided into 2 components. The inner component $\left(r<r_{e}\right)$ follows the stellar distribution without significant need of dark matter $(M / L \leqslant 10)$, while the outer component, consistent with the NFW profile, indicates the dark matter dominance with $M / L$ ratio exceeding 100 at around $10 r_{e}$ for bright galaxies. In a dark-matter dominated cluster A1060, the gravitational potential profile inferred from the X-ray data follows $r^{-1.5}$, i.e. the so-called modified NFW profile, rather than $r^{-1}$.

X-ray observations have revealed dynamical features in the X-ray halo from a number of galaxies. X-ray morphlogies in Chandra image suggest fast gas motion for NGC 4636 and NGC 1404. Hard X-ray emission and X-ray cavities are observed in some groups (e.g. HCG 62), with the latter indicating that non-thermal pressure is overcoming the hot-gas pressure. Therefore, non-thermal processes are causing significant effects in many galaxies. On the other hand, suppression of Fe XVII resonance lines seen in the centers of several galaxies, including NGC 4636, indicates that the gas is not turbulent. Further knowledge about the nature of non-thermal components in galaxies and clusters are important in understanding the evolution of these systems.

X-ray astronomy mission ASTRO-H is planned for launch in 2014 under a major collaboration between Japan and US. This mission will carry microcalorimeters with energy resolution better than $7 \mathrm{eV}$ (about 20 times better than the CCD resolution), with 36 pixels covering a field of view of $3^{\prime} \times 3^{\prime}$. Direct observations of hot-gas motion with $\Delta v \sim 100 \mathrm{~km} \mathrm{~s}^{-1}$ from galaxies and clusters using Doppler shifts of emission lines will be carried out for the first time. Including the capabilities of hard X-ray detectors (multi-layer coated mirrors and Compton cameras), ASTRO-H will be a very powerful mission for the study of non-thermal processes in extended objects.

Keywords. X-rays: galaxies, X-rays: ISM, galaxies: halos 


\section{References}

Fukazawa, Y., Botoya-Nonesa, J. G., Pu, J., Ohto, A., \& Kawano, N. 2006, ApJ 636, 698 Matsushita, K. 2001, ApJ 547, 693

Mathews, W. G. \& Brighenti, F. 2003, ARAA 41, 191 